\title{
Tonsillar Lymphangiomatous Polyps: A Clinicopathologic Series of 26 Cases
}

\author{
David E. Kardon, M.D., Bruce M. Wenig, M.D., Dennis K. Heffner, M.D., Lester D. R. Thompson, M.D. \\ Department of Endocrine and Otorhinolaryngic-Head and Neck Pathology, Armed Forces Institute of \\ Pathology, Washington, DC (DEK, DKH, LDRT), and Montefiore Medical Center, Bronx, New York (BMW)
}

Background: Lymphangiomatous polyps are uncommon benign tumors of the tonsils. Methods: Twenty-six cases of lymphangiomatous polyps diagnosed between 1980 and 1999 were retrieved from the files of the Otorhinolaryngic-Head and Neck Tumor Registry of the Armed Forces Institute of Pathology. Hematoxylin and eosin-stained slides were reviewed to characterize the histologic features of these tumors. Immunohistochemical stains were performed on 15 cases. Clinical follow-up data were obtained. Results: The patients included 13 males and 13 females, ages 3 to 63 years (mean, 25.2 years). Patients experienced dysphagia, sore throat, and the sensation of a mass in the throat. Symptoms were present from a few weeks to years. The tonsillar masses were unilateral in all cases. Clinically, the lesions were frequently mistaken for a neoplasm ( $n=18$ patients). Grossly, all of the lesions were polypoid and measured 0.5 to $3.8 \mathrm{~cm}$ (mean, $1.6 \mathrm{~cm}$ ). Histologically, the polyps were covered by squamous epithelium showing variable epithelial hyperplasia, dyskeratosis, and lymphocytic epitheliotropism. The masses showed a characteristic submucosal proliferation of small to mediumsized, endothelial-lined, lymph-vascular channels lacking features of malignancy. Collagen, smooth muscle, and adipose tissue were present in the stroma. Intravascular proteinaceous fluid and lymphocytes were noted. Immunohistochemical findings confirmed the endothelial origin of the vascular proliferation and a mixed lymphoid population. The differential diagnosis included fibroepithelial

Copyright $\odot 2000$ by The United States and Canadian Academy of Pathology, Inc.

VOL. 13, NO. 10, P. 1128, 2000 Printed in the U.S.A.

Date of acceptance: May 17, 2000.

The opinions or assertions contained herein are the private views of the authors and are not to be construed as official or as reflecting the views of the Department of the Navy or the Department of Defense.

Presented at the United States and Canadian Academy of Pathology Annual Meeting, March 25-31, 2000, New Orleans, Louisiana.

Address reprint requests to: Lester D.R. Thompson, M.D., Department of Endocrine and Otorhinolaryngic-Head and Neck Pathology, 6825 16th Street, NW, Armed Forces Institute of Pathology, Building 54, Room G066-11, Washington, DC 20306-6000; e-mail: thompsonl@afip.osd.mil; fax: 202-782-3130. polyp, lymphangioma, juvenile angiofibroma, and squamous papilloma. In all patients with follow-up, complete surgical excision was curative (mean follow-up, 5.4 years; range, 1 mo to 14 years). Conclusions: We detail the clinical and pathologic features of tonsillar lymphangiomatous polyps. These tumors are uncommon and may clinically be mistaken for a malignant neoplasm. The characteristic histologic features should allow for its correct diagnosis and differentiation from similar appearing tonsillar lesions.

KEY WORDS: Hamartoma, Immunohistochemistry, Lymphangioma, Lymphangiomatous polyp, Pediatric, Polyps, Tonsil.

Mod Pathol 2000;13(10):1128-1133

Benign tumors or tumor-like lesions of the palatine tonsil are less common than malignancies. Squamous papillomas account for the majority of the benign lesions, whereas vascular tumors are reportedly rare (1). We identified ten well-documented cases of lymphangiomatous tumors of the tonsil (MEDLINE 1966-1999) (1-7), with a single case report as early as 1910 (8) (summarized in Table 1). Because of the unusual clinical and pathologic features of these polyps, pathologists and clinicians alike may have difficulty in classifying them correctly. Therefore, we describe the clinical, histopathologic, and immunohistochemical features of a series of 26 lymphangiomatous polyps (LAPs), which are benign lymph-vascular proliferations with varying degrees of fibrous, adipose, and lymphoid components.

\section{MATERIALS AND METHODS}

Twenty-six cases of lymphangiomatous polyps were identified in the files of the OtorhinolaryngicHead and Neck Pathology Registry of the Armed Forces Institute of Pathology from 1980 to 1999, chosen from a total of 1389 (1.9\%) tonsillar benign and malignant tumors. Two of the cases in our series have been previously reported (3). The lan- 


\begin{tabular}{|c|c|c|c|c|c|c|}
\hline $\begin{array}{c}\text { No. } \\
\text { of } \\
\text { Cases }\end{array}$ & Author (Ref.) & Year & Age & Gender & Presenting Symptoms & Diagnosis \\
\hline 1 & Goris (8) & 1910 & $\mathrm{nr}$ & M & Dysphagia and dyspnea & Cavernous lymphangioma \\
\hline 2 & Menzel (17) & 1919 & $\mathrm{nr}$ & $\mathrm{Nr}$ & Acute tonsillitis & Lymphangioma \\
\hline 3 & Harrison (1) & 1960 & 27 & $\mathrm{~F}$ & Sore throat, 'spitting blood" & Lymphangioma \\
\hline 4 & Ash (18) & 1962 & 20 & M & Frequent sore throat & Hamartoma \\
\hline 5 & Visvanathan (2) & 1971 & 31 & M & Dysphagia for solids, sore throat & Pedunculated lymphangioma \\
\hline 6 & Araujo (19) & 1977 & 18 & M & Acute tonsillitis, mass & Lymphangioma \\
\hline 7 & Al Samarrae (3) & 1985 & 32 & $\mathrm{M}$ & Sore throat, mass & Polypoid lymphangioma \\
\hline 8 & & & 35 & $\mathrm{~F}$ & Slowing deglutition & Polypoid lymphangioma \\
\hline 9 & Abu Shara (6) & 1991 & 41 & M & Dysphagia, 'lump' in throat & Hamartomatous polyp \\
\hline 10 & Roth (7) & 1996 & 14 & M & Recurrent tonsillitis, mass & Lymphangiomatous polyp \\
\hline
\end{tabular}

nr, not reported; M, male; F, female

guage in the literature used to diagnose this entity is variable and includes variants of the terms "polypoid," "lymphangiomatous," and "hamartomatous." Materials within the Institute's files were supplemented by a review of patient demographics, symptoms at presentation, operative and surgical pathology reports, and information obtained from written questionnaires or oral communication with the treating physician(s). Fifteen cases were obtained from civilian sources, including university medical centers and foreign contributors, and 11 cases were obtained from military hospitals. This clinical investigation was conducted in accordance and compliance with all statutes, directives, and guidelines of the Code of Federal Regulations, Title 45, Part 46, and the Department of Defense Directive 3216.2, relating to human subjects in research.

We reviewed the hematoxylin and eosin-stained (H\&E) slides and evaluated the histologic features. Lesional fibrosis was graded on a scale from 0 to $3+$ based on the amount and type of collagen. Although the diagnosis of tonsillar LAP does not require ancillary stains, we performed a battery of immunohistochemical stains on formalin-fixed, paraffin-embedded, $4-\mu$ m-thick tissue sections to characterize the antigenic profile of the entity. Immunohistochemical stains were performed on a single block in 15 cases using the standardized avidin-biotin-peroxidase method as has been pre- viously described (9). The antibodies and titers used are summarized in Table 2. Standard positive controls were used throughout, with normal serum used as the negative control.

\section{RESULTS}

\section{Clinical Demographics and Presentation}

The 13 males and 13 females ranged in age from 3 to 63 years, with a mean age at presentation of 25.2 years (median, 25.5 years). There was no difference in age at presentation between females (25.0 years) and males (25.5 years). Eight of the patients were considered pediatric or adolescent (less than 21 years).

Twenty patients presented clinically with a tonsillar mass. Patients also presented with dysphagia, sore throat, and/or difficulty swallowing. Two patients presented with symptoms of tonsillitis alone. In all cases the LAP was unilateral, without a side predilection. One patient presented with the mass in his nasopharynx, arising from the adenoids rather than the faucial tonsils.

\section{Clinical Management and Outcome}

Fifteen patients were treated by excisional biopsy of the mass and 11 by tonsillectomy. No additional

TABLE 2. Summary of Immunohistochemical Antibodies

\begin{tabular}{|c|c|c|c|c|}
\hline Antibody & $\begin{array}{l}\text { Primary } \\
\text { Antibody }\end{array}$ & Company & Dilution & Antigen Unmasking \\
\hline Smooth muscle actin & $\mathrm{mm}$ & $\begin{array}{l}\text { Sigma ImmunoChemicals } \\
\text { (St. Louis, MO) }\end{array}$ & $1: 8000$ & None \\
\hline Factor-VIII-related antigen & $\mathrm{rp}$ & DAKO (Carpenteria, CA) & $1: 800$ & ${ }^{a}$ Protease treatment \\
\hline CD31 & $\mathrm{mm}$ & DAKO & $1: 40$ & None \\
\hline CD34 & $\mathrm{mm}$ & $\begin{array}{l}\text { Biogenex Laboratories (San } \\
\text { Ramon, CA) }\end{array}$ & $1: 80$ & ${ }^{a}$ Protease treatment \\
\hline CD45-RB (LCA) & $\mathrm{mm}$ & DAKO & $1: 200$ & None \\
\hline CD3 & $\mathrm{rp}$ & DAKO & $1: 500$ & ${ }^{a}$ Protease treatment \\
\hline CD20 (L26) & $\mathrm{mm}$ & DAKO & $1: 200$ & None \\
\hline
\end{tabular}

mm, mouse monoclonal; rp, rabbit polyclonal.

${ }^{a}$ Antibody treatment involved predigestion for $3 \mathrm{~min}$ with $0.05 \%$ Protease VIII (Sigma Chemical Co., St. Louis, MO) in a $0.1 \mathrm{M}$ phosphate buffer at a $\mathrm{pH}$ of 7.8 at $37^{\circ} \mathrm{C}$. 
treatment was rendered for this lesion in any of the patients. Follow-up information was available in 23 cases. We did not obtain additional follow-up for the three foreign patients, two of which were previously reported (3). There was no tonsillar abnormality or development of recurrent disease in any patient during the follow-up period that ranged from 1 mo to 14 years (mean, 5.4 years). All patients were alive without evidence of disease at last follow-up with the exception of one patient who died of unrelated causes after 5.8 years.

\section{Pathology Findings \\ Macroscopic findings}

The tumors ranged in greatest dimension from $0.5 \mathrm{~cm}$ to $3.8 \mathrm{~cm}$ (mean, $1.6 \mathrm{~cm}$ ). They were described as polypoid (Fig. 1) with a smooth white, tan, or yellow cut surface. The masses varied from spongy to firm and fibrous. Sixteen of the lesions were conspicuously pedunculated or polypoid, whereas the remaining ten were sessile to unremarkable.

\section{Microscopic findings}

Histologically, the overlying surface epithelium was either squamous or respiratory, and in a few cases was hyperplastic or dyskeratotic (although without dysplasia). Three cases showed a prominent nested epitheliotropism, in which small lymphocytes (round nuclei and condensed nuclear chromatin) were packed into rounded intramucosal spaces (Fig. 2). The presence of lymphocytes within tonsillar epithelium is normal. The lymphocytes are usually arranged in an intimate affiliation of individual lymphocytes percolating between the epi-

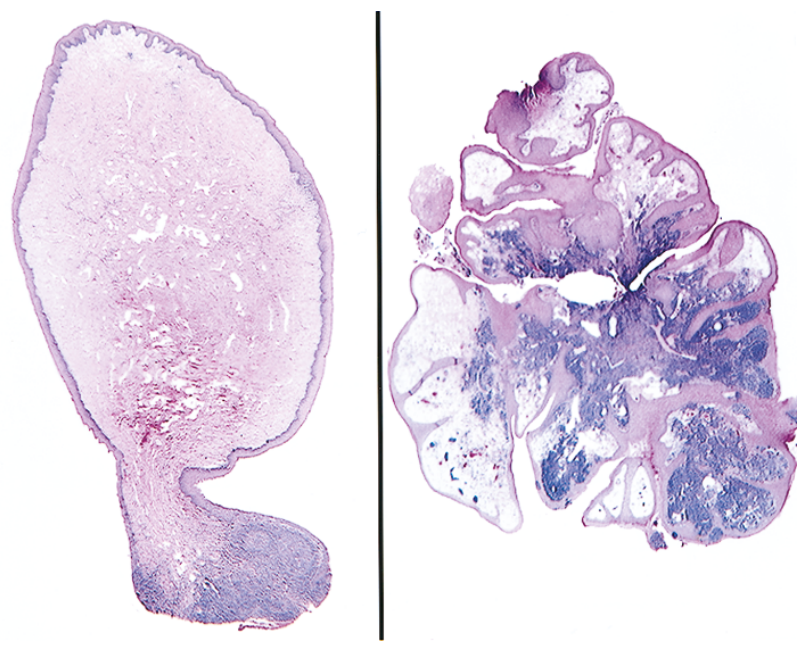

FIGURE 1. Typical appearance of a tonsillar lymphangiomatous polyp. The lesion is pedunculated and composed of a proliferation of vascular channels within an abundant fibrous stroma (left, original magnification, $20 \times$ ). Some of the lesions had a more papillary architecture and minimal stromal fibrosis (right, original magnification, $20 \times$ ). thelial cells of the crypt surface epithelium forming a classic "lymphoepithelium." Therefore, the formation of discrete pockets of lymphocytes in LAP is unusual, and to the authors' knowledge not previously described. The significance and etiology of this epitheliotropism is unclear.

LAPs are composed of a proliferation of submucosal dilated lymphatic vascular channels and varying amounts of fibrous connective tissue. In accordance with the macroscopic appearance, the majority of the tumors were pedunculated polypoid masses arising from the surface of the tonsil (Fig. 1) and showed no infiltration of the underlying stroma. Four of the lesions had a more papillary architecture, coupled with the lymphoid and edematous stroma. None of the cases extended into the surrounding pharyngeal tissues.

The vascular components were thin-walled and usually contained proteinaceous fluid ( $n=19$ cases) and lymphocytes ( $n=20$ cases). In a few cases, the lymphocytes were packed into the vessels in such as way as to make the channels difficult to identify on routine H\&E stain (Fig. 3). A stromal adipose tissue component was present in nine cases (Fig. 4). Fibrosis was prominent ( 2 to $3+$ ) in 18 cases, minimal $(1+)$ in four, and absent in the remaining four.

\section{Special procedures}

Factor VIII-related antigen was positive in the endothelium and subendothelium of the lymphatic channels in all $(n=15)$ cases tested (Fig. 3). The vessels were reactive with anti-CD31 in 13 cases, anti-CD34 in six cases, and one case was negative for both markers. Smooth muscle actin was present in the walls of the dilated lymphatic vessels in all of the cases tested. The hematologic markers (CD45RB [LCA], CD3 [T-cell] [Fig. 3], and CD20 [L26, B-cell]) were expressed in the lymphoid cells

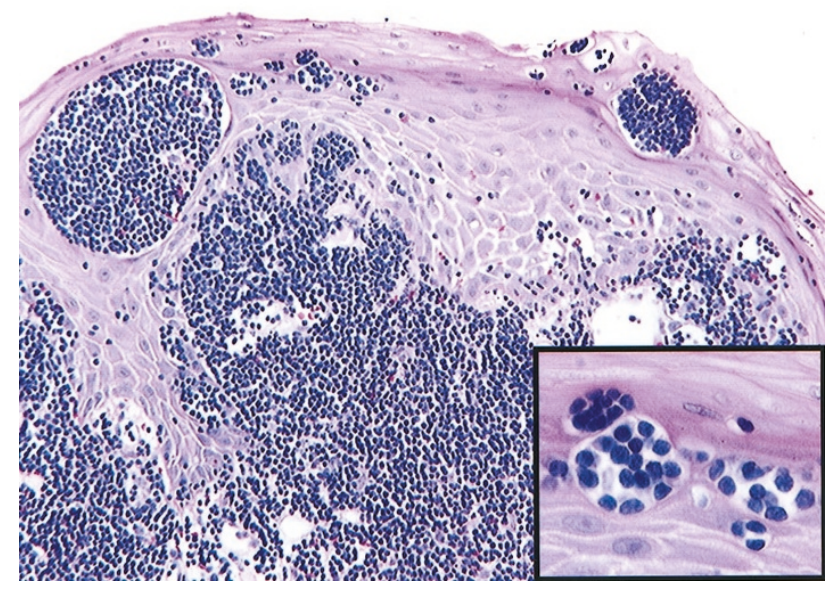

FIGURE 2. Nested epitheliotropism of lymphocytes seen at the surface of some LAPs (original magnification, $200 \times$ ). There is no cytologic atypia (inset, original magnification, $600 \times$ ). 


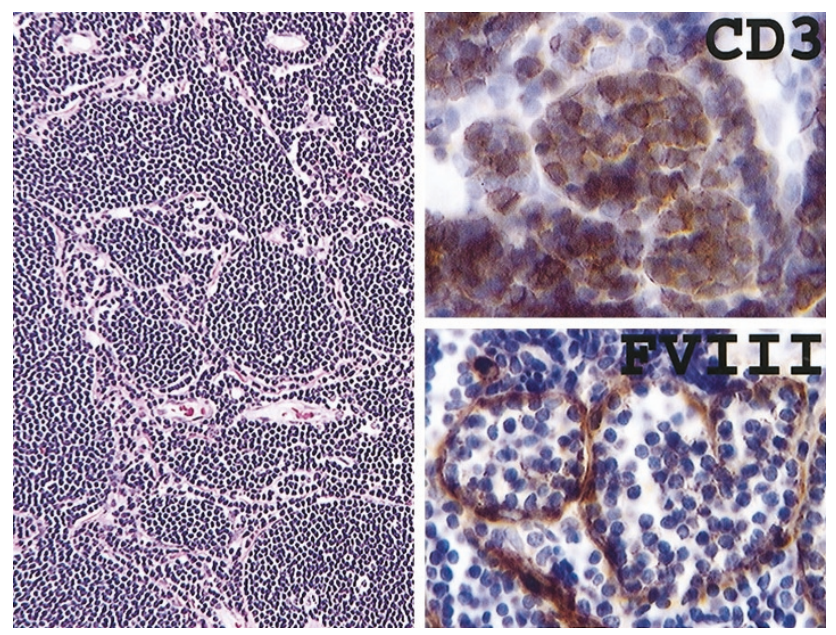

FIGURE 3. The lymphatic channels are stuffed with lymphocytes and more difficult to identify (left; original magnification,100×). A predominantly T-cell (CD3) immunophenotype of the intravascular lymphocytes (top right, original magnification, $600 \times$ ). Factor VIII-related antigen highlights the endothelium of the lymphatic channels (bottom right, original magnification, $600 \times$ ).

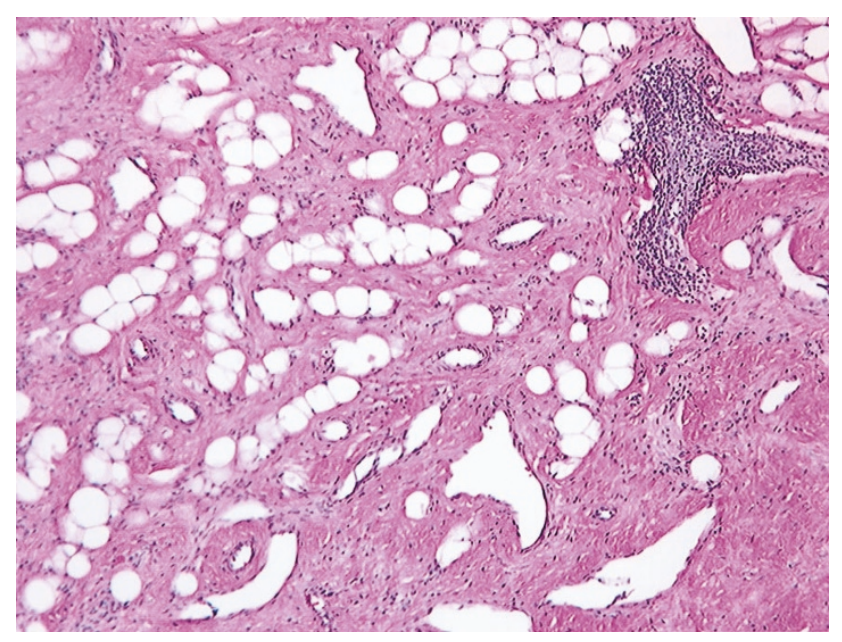

FIGURE 4. The three components of tonsillar lymphangiomatous polyps: dilated lymphatic channels, fibrous, and/or adipose stroma, and lymphoid tissue (original magnification, $100 \times$ ).

in an expected pattern of distribution for tonsillar tissue in all cases. Lymphocytes within the vascular channels and within the epithelium of the polyps were predominantly CD3 immunoreactive, even though there was a polymorphous B-cell and T-cell immunophenotype.

\section{DISCUSSION}

The head and neck are the most common anatomic regions for lymphangiomatous lesions, accounting for over $90 \%$ of all lymphangiomas (10). Most arise in the skin and subcutaneous tissues, but other sites include the larynx, parotid gland, mouth, and tongue (11). The tonsil is a less common site for the development of lymphangiomatous tumors, and their classification in this location is confusing. In the early part of the 20th century, histologically identical lesions of the tonsil were reported by a number of different names, including angiomas (12), angiofibromas, or fibroangiomas (13). Still others have had difficulty classifying their cases specifically, and have named them fibrolipomas after the stromal components, or have given them a more descriptive diagnosis, such as "polypoid tumor containing fibroadipose tissue." (3)

Consequently, the true incidence of these lesions is difficult to accurately assess from the literature. As such, we share the opinions of others $(1,3)$ and believe lymphangiomatous tonsillar tumors have a higher incidence than is reported. Furthermore, they are probably more common than other angiomatous lesions in this location. In our series, lymphangiomatous polyps accounted for $1.9 \%$ of all tonsillar tumors seen in consultation during the same period of review. In a previous study (14), lymphangiomatous lesions represented $8 \%$ of all benign tonsillar tumors, whereas hemangiomas and fibromas represented 2 and 3\%, respectively.

We prefer the term lymphangiomatous polyp rather than lymphangioma because the pathogenesis of these lesions is still unclear. As noted in the literature (5), the lymphatic channels of LAPs are frequently dilated, but generally are not as prominent as in the typical lymphangioma. In addition, the stromal components are frequently more abundant than the vessels. We agree with the assertion that these lesions are most likely hamartomatous $(5,6,15)$ because they consist of a haphazard proliferation of elements that are normally found in the tonsil. These tumors occasionally become large and cause obstructive symptoms and in these circumstances, have generally been present for years-up to 30 years in one reported case (6).

Histologically, four of our lesions were papillary and marked a close resemblance to the previously reported "lymphoid papillary hyperplasia" or "papillary lymphoid polyp" of the tonsil $(4,16)$. This rare entity occurs exclusively in children. Although some of our cases were papillary, they did not have the prominent lymphoid follicles characteristic of that diagnosis, and instead had more prominent lymphatic spaces (Fig. 5). LAPs are separated from the tonsillar parenchyma, whereas papillary hyperplasia blends with the underlying lymphoid stroma. Moreover, 18 of our cases did not occur in pediatric-aged patients. Given that these two lesions contain similar components in differing architectures and proportions, it is quite possible they may represent a forme fruste of the same entity along a developmental arc.

As previously noted, immunohistochemistry is not needed to definitively diagnose LAP. However, in the interest of specifically documenting the immunoprofile of these lesions in a large series, we 


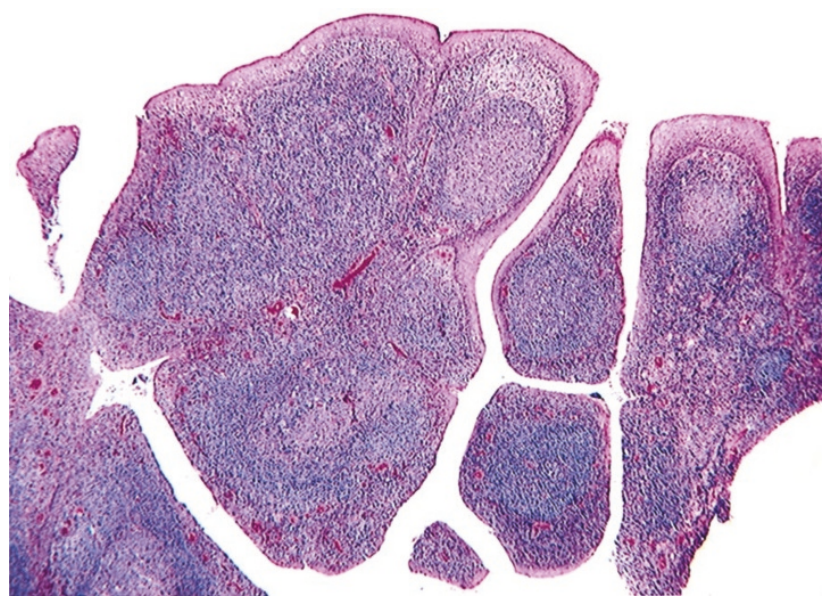

FIGURE 5. Lymphoid papillary hyperplasia of the tonsil: papillary architecture with prominent germinal centers and no appreciable lymphatic vascular component (original magnification, 40×).

applied a battery of commercially available immunoantibodies. As expected, the dilated lymphatic vessels contain at least a thin wall of smooth muscle, and are uniformly reactive with Factor VIIIrelated antigen. CD31 is more frequently reactive than CD34, although this difference in staining did not correlate with other features of the tumors. The endothelial markers may be of use in highlighting the vessels in cases that have abundant intraluminal lymphocytes expanding the channels to the point they may be difficult to recognize. Notably, the endothelial markers were non-reactive in the intramucosal spaces that surrounded the nested epitheliotropic lymphocytes. The hematologic markers showed the expected pattern of distribution for B-cells and T cells. Most of the lymphocytes, including the lymphocytes, were of T-cell origin (Fig. 2). The lymphocyte morphology was that of normal mature lymphocytes and as such should not be confused with the similar migration into epithelium, so-called "Pautrier's microabscesses," which may be seen in malignant T-cell lymphoma.

The differential diagnoses of contributing pathologists included juvenile angiofibroma, fibroepithelial polyps, papilloma, and lymphangioma. It is important to distinguish LAP from juvenile angiofibroma as the latter lesion is usually treated more aggressively to prevent possible recurrence. Clinically, angiofibromas typically occur in the nasopharynx of adolescent males, often attaining a large size, with extensive growth and even bone erosion, and presenting with epistaxis due to the rich blood supply. Although one of our cases of LAP occurred in the nasopharynx, it developed in a 28year-old female without epistaxis and did not invade into the surrounding structures. Histologically, the stroma of angiofibromas is more cellular, composed of stellate and plump cells, and contains staghorn-like thin walled vascular channels. LAPs usually have a relatively paucicellular fibrous background and many more lymphocytes. Squamous papilloma is usually an exophytic surface epithelial proliferation, arranged in multiple layers, not invading the underlying stroma, and lacking a lymphatic and lymphocytic component. When lymphangiomas are described, they usually contain widely dilated vascular channels with luminal proteinaceous fluid and lymphocytes. Our cases of LAP contained dense fibrous connective tissue to a variable degree, a rich lymphocyte investment, and a number contained adipocytes. These components are the normal constituents of this site, except arranged in a different pattern, and so we believe they more likely represent a hamartomatous proliferation rather than being a typical lymphangioma.

\section{Summary}

Lymphangiomatous polyps of the tonsil are benign tumors that most frequently present as mass lesions and are composed of dilated lymphatic channels amid a fibrous, lymphoid, and/or adipose stroma. They are most likely hamartomatous proliferations, and as such are cured by simple surgical excision. After surgical resection, there were no incidences of recurrence. They (LAPs) have a varied histology and may exhibit papillary growth. LAPs are probably not as rare as reported in the literature, and accounted for approximately $2 \%$ of all tonsillar neoplasms in our study.

Acknowledgment: The authors thank Ms. Serena Lei for her critical review of the article.

\section{REFERENCES}

1. Harrison G, Johnson L. Lymphangioma of the tonsil. Ann Otol Rhinol Laryngol 1960;69:961-8.

2. Visvanathan PG. A pedunculated tonsillar lymphangioma. J Laryngol Otol 1971;85:93-6.

3. Al Samarrae SM, Amr SS, Hyams VJ. Polypoid lymphangioma of the tonsil: report of two cases and review of the literature. J Laryngol Otol 1985;99:819-23.

4. Pyun KS, Friedman SI. Papillary lymphoid polyp of the palatine tonsil. Ear Nose Throat J 1985;64:243-5.

5. Heffner DK. Pathology of the tonsils and adenoids. Otolaryngol Clin North Am 1987;20:279-86.

6. Abu Shara K, Al-Muhana A, Al-Shenawy M. Hamartomatous tonsillar polyp. J Laryngol Otol 1991;105:1089-90.

7. Roth M. Lymphangiomatous polyp of the palatine tonsil. Otolaryngol Head Neck Surg 1996;115:172-3.

8. Goris. Lymphangiome cavernaux de l'amygdale. Ann Soc Belge Chi 1910;8:307-9.

9. Hsu SM, Raine L, Fanger H. Use of avidin-biotin-peroxidase complex (ABC) in immunoperoxidase techniques: a comparison between $\mathrm{ABC}$ and unlabeled antibody (PAP) procedures. J Histochem Cytochem 1981;29:577-80.

10. Kennedy TL. Cystic hygroma-lymphangioma: a rare and still unclear entity. Laryngoscope 1989;99:1-10.

11. Stal S, Hamilton S, Spira M. Hemangiomas, lymphangiomas, 
and vascular malformations of the head and neck. Otolaryngol Clin North Am 1986;19:769-96.

12. Ormerod F. Angioma of the tonsil. J Laryngol Otol 1926;41: 797-800.

13. Hara H. Benign tumors of the tonsil. Arch Otol 1933;18:62-9.

14. Hyams VJ. Differential diagnosis of neoplasia of the palatine tonsil. Clin Otolaryng 1978;3:117-26.

15. Ash J, Raum M. Tonsils. In: Ash J, Raum M, editors. An atlas of otolaryngic pathology. Washington, DC: American Registry of Pathology;1956. p. 286, 298.

16. Carrillo-Farga J, Abbud-Neme F, Deutsch E. Lymphoid pap- illary hyperplasia of the palatine tonsils. Am J Surg Pathol 1983;7:579-82.

17. Menzel H. Lymphangioms der linken Tonsille. Monatsschrift Der Ohrencheil 1919;53:509.

18. Ash J, Beck M, Wilkes J. Mesodermal neoplasms. In: Ash J, Beck M, Wilkes J, editors. Tumors of the upper respiratory tract and ear. Atlas of tumor pathology. 1st ed. Fasc 12. Washington, DC: Armed Forces Institute of Pathology, 1962. p. $140-1$.

19. Araujo F. Lymphangiome de l'amygdale palatine. Ann Otolaryngol Chir Cervicofac 1977;94:111-6.

\section{Book Review}

\section{Rosen PP: Breast Pathology. Diagnosis by Nee- dle Core Biopsy, 311 pp, Philadelphia, Lip- pincott Williams \& Wilkins, 1999, (\$169).}

This well-illustrated manual deals with the histologic features of breast diseases as seen in needle core biopsies. Because the use of needles of various calibers is becoming more and more popular, the need for such books will obviously increase, and they might turn out to be popular with practicing pathologists.

The book is divided into 31 chapters that cover essentially all features of normal and abnormal breast biopsies encountered in a busy hospital practice. Color figures illustrating these conditions are excellent, even though some of them are accompanied with rather brief descriptions. The text is also short and to the point. Obviously the book was aimed at experienced pathologists who will have no problems identifying the salient features in these illustrations and will not need a more detailed text. The beginners probably would be better off with Dr. Rosen's "big book."

In addition to common entities, the book also deals with the more esoteric lesions, in- cluded for the sake of completeness. These entities need to be considered in the differential diagnosis of the more common diseases, and to better understand the variants that may pose diagnostic problems. Less experienced pathologists, such as the present reviewer, probably would have profited if some differential diagnostic points were spelled out in greater detail, but even so I think that I have learned a lot, and especially about the differences between the histopathology of tumors in conventional and needle core biopsies.

I have kept Dr. Rosen's book next to my microscope and have consulted it successfully in working up some complex breast biopsies. The high quality pictures were invariably informative and are my favorite feature-I am almost sure that they will be appealing to other pathologists as well. The book is ideally suited for busy hospital-based practicing pathologists in need of an authoritative and reliable manual.

Fang Fan

University of Kansas School of Medicine Kansas City, Kansas 